\title{
Measurement of complementary strain fields at the grain scale
}

\author{
Wang $\mathrm{CHOW}^{1,}$, Denis SOLAS ${ }^{2, \mathrm{~b}}$, Guillaume PUEL ${ }^{1, c}$, Eric PERRIN ${ }^{1, d}$, \\ Thierry BAUDIN ${ }^{2, e}$ and Veronique AUBIN ${ }^{1, f^{*}}$ \\ ${ }^{1}$ Ecole Centrale de Paris, MSSMat, UMR CNRS 8579, F-92296 Chatenay-Malabry, France \\ 2Univ Paris Sud, ICMMO, UMR 8182, F-91405 Orsay, France \\ awang.chow@ecp.fr, bdenis.solas@u-psud.fr, 'cguillaume.puel@ecp.fr, deric.perrin@ecp.fr, \\ ethierry.baudin@u-psud.fr, ${ }^{f}$ veronique.aubin@ecp.fr *
}

Keywords: Diffraction of monocrystal, Digital image correlation, aluminium alloy.

\begin{abstract}
In this study, a monotonous mechanical test is performed on a sample in Al-alloy containing about twenty-five grains. During this test, both the total and elastic intragranular strain fields are measured at the same time. The former field is observed by digital image correlation technique while the latter one is measured by X-ray diffraction. The strain fields obtained are then dedicated to identify the parameters of a crystalline constitutive model, using finite element simulation.
\end{abstract}

\section{Introduction}

The crystalline plasticity models are broadly used to predict the mechanical behaviour of crystalline materials. As they are based on the strain-causing physical mechanisms, these equations are able to deduce the microstructure changes of a material under complex loadings [1-4]. It can predict, for example, local disorientation due to hot forming or the plastic strain localisation which induces fatigue crack initiation.

However, the parameters of these models are difficult to identify. This procedure is generally carried out using only data at the macroscopic scale, which cannot take into account the range of heterogeneities of the grain scale. Therefore, the objective of this research is to develop a robust procedure to identify this type of model using the grain scale information. Two aspects are then developed in parallel: measuring the total and elastic strain fields and simulating a crystalline aggregate in order to identify the parameters of the behaviour model. The surface fields' information will then be used for the identification of material parameters of the crystalline plasticity constitutive equations [5].

\section{Objective}

The objective is to measure simultaneously both the total $\varepsilon^{\mathrm{t}}$ and elastic $\varepsilon^{\mathrm{e}}$ strain fields at each loading level imposed during an in-situ tensile test (Fig. 1). This test is conducted on a sample in aluminium alloy with around twenty-five grains. The test is stopped at increasing levels of strain, and total and elastic strain fields measured in the useful part of the specimen. The total strain field is observed by digital image correlation (DIC) while the elastic one is measured by X-ray diffraction (XRD).
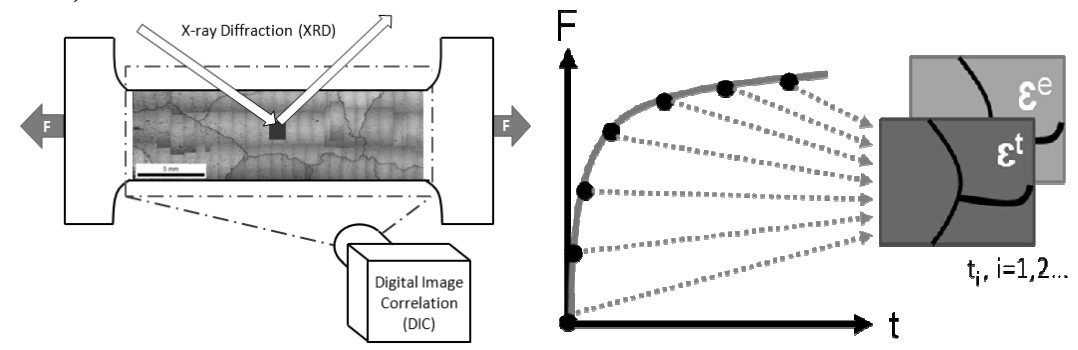

Figure 1. Principle of in-situ simultaneous kinematic full-fields measurements during a tension test 


\section{Material and sample preparation}

During the experiments, a specimen of aluminium alloy (5052) is used. The chemical composition is given in table 1 .

Table 1. Chemical composition of the aluminium alloy used (5052).

\begin{tabular}{|l|l|l|l|l|l|l|c|}
\hline $\mathrm{Si}$ & $\mathrm{Fe}$ & $\mathrm{Cu}$ & $\mathrm{Mn}$ & $\mathrm{Mg}$ & $\mathrm{Cr}$ & $\mathrm{Zn}$ & $\mathrm{Al}$ \\
\hline 0.45 & 0.45 & 0.10 & 0.10 & $2.2-2.8$ & $0.15-0.35$ & 0.10 & Balance \\
\hline
\end{tabular}

The specimen dimensions are $13 \times 43 \mathrm{~mm}^{2}$ with a thickness of $0.5 \mathrm{~mm}$ (Fig. 2a). To facilitate the measurements of strain gradients inside the grains, a specimen with very large grains has been chosen. The procedure of recrystallization and grain growth was optimized in order to obtain grain sizes of about $5 \mathrm{~mm}$.

a

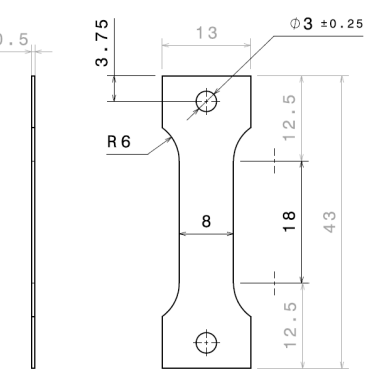

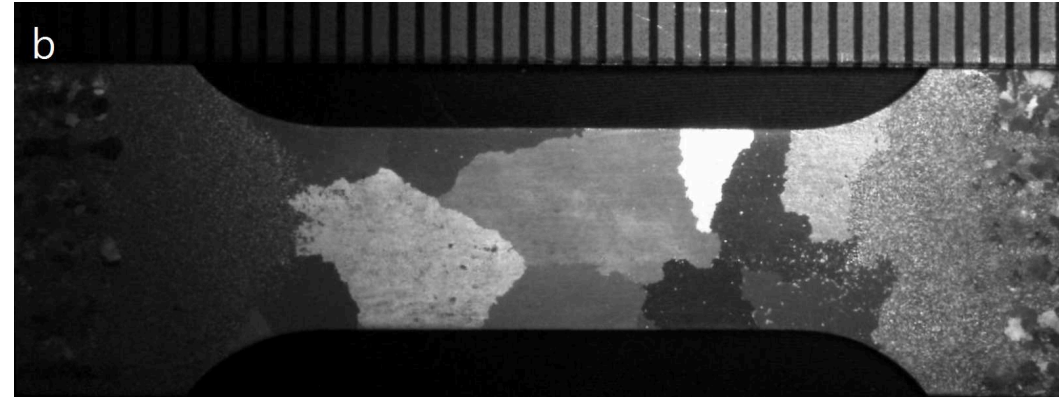

Figure 2. a. Sample dimensions [mm], b. Sample with large grains.

With the aim of obtaining multicrystalline structure, the sample is first subjected an annealing at $450{ }^{\circ} \mathrm{C}$ for two hours to release internal residual stresses. Critical work hardening at $4.7 \%$ strain is carried out to generate recrystallisation nucleuses. Finally, recrystallisation annealing is performed at $540^{\circ} \mathrm{C}$ for two days to obtain large grains (Fig. 2b).

\section{Experimental Methods}

\section{Simultaneous $\varepsilon^{\mathrm{e}}$ and $\varepsilon^{\mathrm{t}}$ measurements}

Implementation

The micro-tensile machine (MICROTEST) was designed by Deben for use within a SEM. Its XRD mounting system allows performing mechanical test inside the X-ray diffraction machine. Considering a suitable load cell and calibration system for this apparatus, the maximum tensile loading is $1 \mathrm{kN}$. For these reason, the sample dimension and its materials should be carefully optimized.

As X-Ray measurements take a very long time (several days), it is very important that the machine ensures a precise constant loading during the entire X-Ray scan. To qualify the machine, a relaxation and a creep test were conducted independently. By maintaining the distance of the clips constant (31.05mm) for 5 days, the distance variation between the clips was about $1 \mu \mathrm{m}$. The force dropped considerably $(78.87 \mathrm{~N})$ during the first 40 minutes then decreased slowly $(43 \mathrm{~N})$ for the rest of time. While keeping constant force of the clips at $600 \mathrm{~N}$ for 5 days, the force variation was around $1 \mathrm{~N}$. The distance variation between the clips was about $5 \mu \mathrm{m}$.

The X-ray diffraction measurement is performed on a X'Pert Pro MRD 7-axis (x, y, z, $\psi, \varphi, \omega$ and $2 \theta$ ) goniometer by Panalytical. Starting from the cobalt source tube operating at $45 \mathrm{kV} / 40 \mathrm{~A}$, the $\mathrm{X}$-ray beam first passes through a $\beta$-filter in Iron to sort out all the $\alpha$-wavelengths. A poly-capillary lens with a diameter of $8 \mathrm{~mm}$ is then needed to convert a highly divergent beam of X-rays into a quasi-parallel beam of low divergence. The beam size is limited by a knob adjustable crossed slit collimator before diffracting the sample positioned on the wafer holder. The diffracted beams go through a Soller slit holder with vertical and horizontal blades spaced $0.5 \mathrm{~mm}$ and are finally quantified by a Xenon filled gaseous proportional detector. 

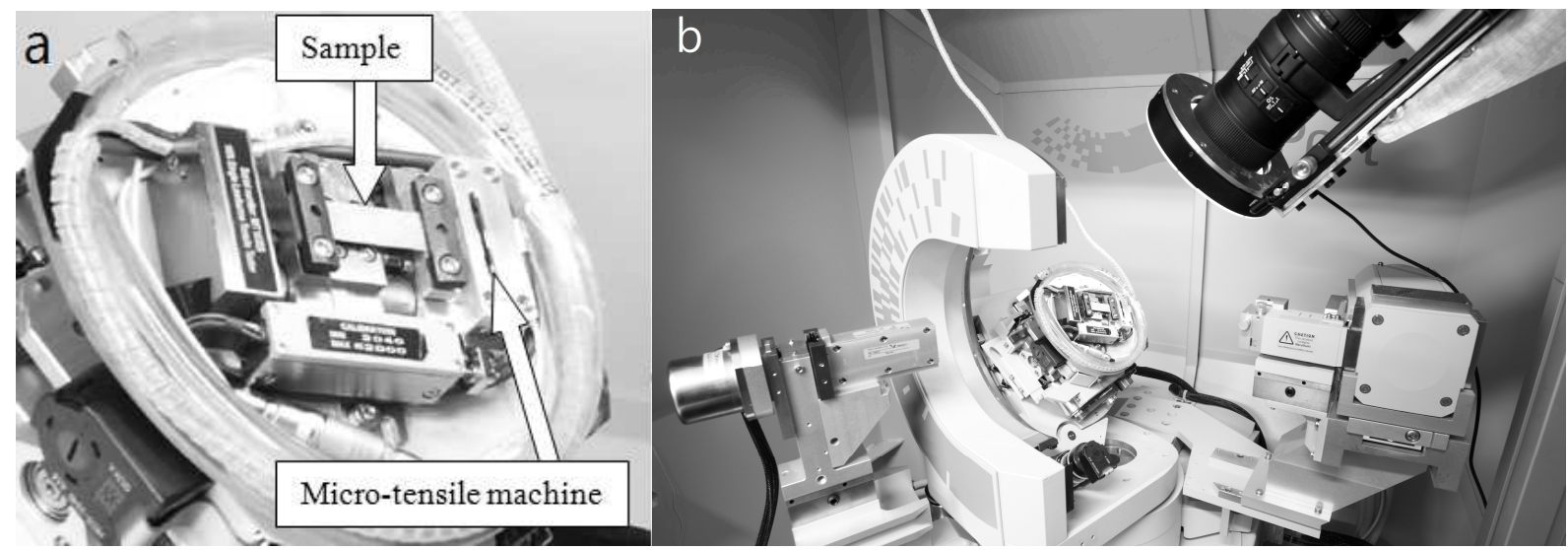

Figure 3. a. Sample inside the micro-tensile machine. b. Assembly of the setup.

The micro-tensile machine and a single-lens reflex camera are installed inside the XRD machine (Fig. 3b). To integrate all the elements inside the diffractometer, the micro-tensile machine is positioned on the cradle of the goniometer by considering its weight and the distance between sample surface and X-ray beams (z-axis). The reflex camera faces the specimen vertically. A cable guide has been designed around the micro-tensile machine for tidying up its power cable and preventing it crossing the beam during diffraction measurements.

\section{Mesh of the sample}

The grain position on the sample (Fig. 2b) is captured by an optical microscope. A mesh size is defined according to the grain size. There are approximately 20 points of XRD measurement in a grain. In our case, the useful part of the sample is $21 \times 6 \mathrm{~mm}^{2}$ and the mesh size is $1 \times 1 \mathrm{~mm}^{2}$ (Fig. 4). The spatial resolution for CID is much finer than for XRD. It will be presented and defined in later.

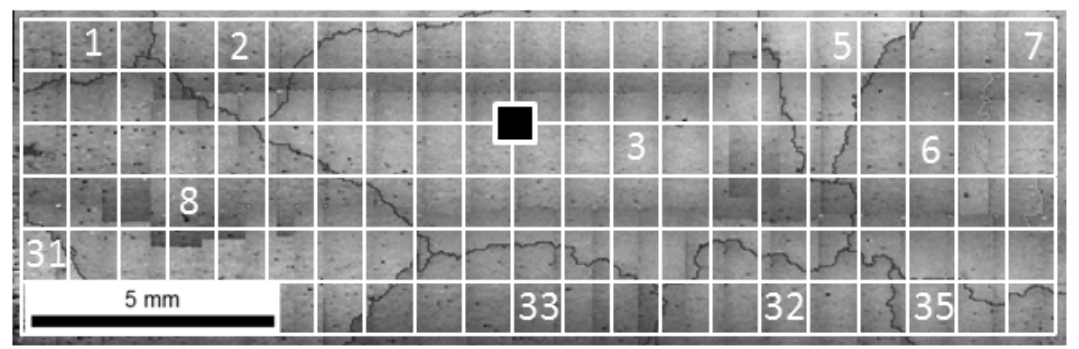

Figure 4. A meshed sample with its grains numbered. The black-block indicates the measuring position of X-ray beam.

\section{Initial Microstructure}

The crystallographic orientation

Crystal orientations $\left(\varphi_{1}, \Phi, \varphi_{2}\right)_{\text {grain }}$ can be determined from the position of the diffraction peaks. First, a complete texture scanning is performed on each single crystal on the sample in its initial state. The peak positions $(\psi, \varphi)_{\{h k l\}}$ in the pole figure of $\{220\}_{\text {grain }}$ and $\{222\}_{\text {grain }}$ inter-reticular plans are identified (Fig. 5a).

After that, Euler angle triplets are generated every $30^{\circ}$ and the corresponding $(\psi, \varphi)_{\{220\}}$ and $(\psi$, $\varphi)_{\{222\}}$ are calculated. By minimizing the difference between the calculated and measured $(\psi, \varphi)$ values, Euler angles of each grain can then be deducted.

Once the crystal orientation is identified, all the resting $(\psi, \varphi)_{\{h k l\}}$ can then be calculated (Fig. 5b). Unlike EBSD scanning which requires a high quality of polishing on sample surface, this is a non destructive method to determine the materials texture. Since obtaining a sufficiently precise pole figure for one $\{h k l\}$ plan requires 9-hour scanning, the induction process can avoid the bulky scanning for other $\{h k l\}$ plans (Fig. 5b). The initial orientations of each crystal could be used for numerical simulation afterward. 

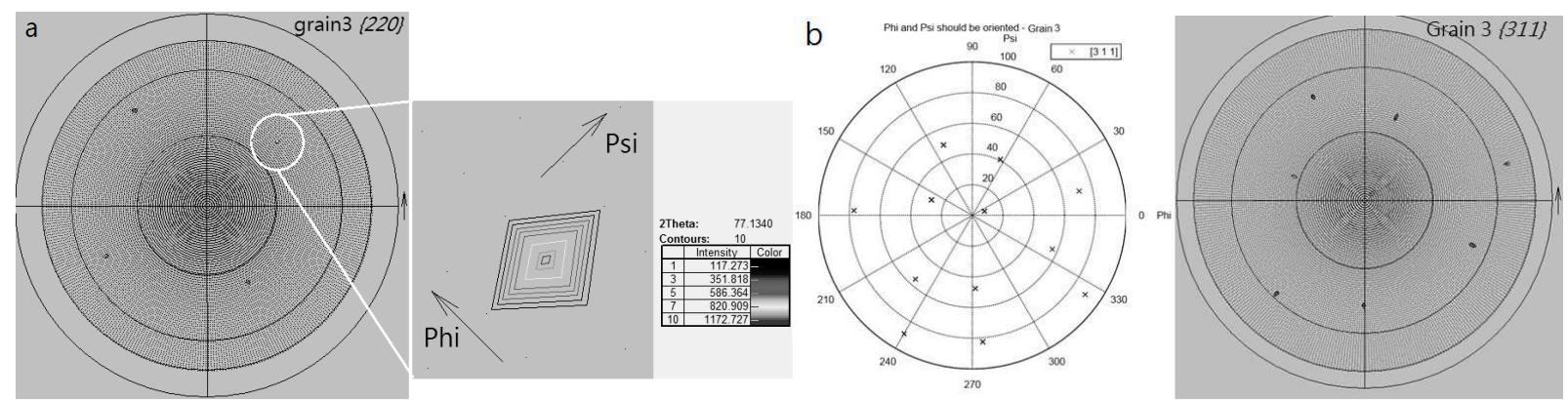

Figure 5. Grain 3: a. Pole figure of $\{220\}$ plans. b. Calculated and measured Pole figure of $\{311\}$ according to crystal orientation.

Optical Microscope

With the help of optical microscope, each grain position is recognized (Figure 4). It facilitates the XRD scanning programming as well as Finite Element meshing.

\section{$\varepsilon^{\mathrm{e}}$ field measurement}

X-ray diffraction technique: First Ortner Method [6]

The metric tensor $\mathrm{G}$ can be calculated during a tensile test with $(\psi, \varphi)_{\{h k l\}}$, so as the strain tensor $\varepsilon$ can be deduced. The relationship between selected $\{h k l\}$ plans distance (vector $\mathrm{D}^{*}$ in the reciprocal lattice) and reciprocal metric tensor $\mathrm{G}^{*}$ can be described with a matrix $\mathrm{H}$, which is constructed by the coordinates of the diffraction vectors of these $\{h k l\}$ plans in the reciprocal lattice (Eq.1).

$$
\mathrm{HG}^{*}=\mathrm{D}^{*}
$$

By the method of least squares, $\mathrm{G}^{*}$ can be calculated with at least the $6\{\mathrm{hkl}\}$ plans measured.

$$
\mathrm{G}^{*}=\left(\mathrm{H}^{\mathrm{T}} \cdot \mathrm{H}\right)^{-1} \mathrm{H}^{\mathrm{T}} \cdot \mathrm{D}^{*}
$$

The advantage of this method is its independence regarding to the $2 \theta$ rotation.

Then, the strain tensor $\varepsilon$ is determined by:

$$
\varepsilon_{i j}=\mathcal{E}^{i j}=\frac{g^{\mathrm{ij}}-g_{0}^{i j}}{2 \sqrt{\mathrm{g}_{0}^{\mathrm{ii}}} \sqrt{\mathrm{g}_{0}^{\mathrm{jj}}}}
$$

where $\mathrm{g}_{0}{ }^{\mathrm{ij}}$ et $\mathrm{g}^{\mathrm{ij}}$ are the contravariance of the initial and the deformed metric tensor $\mathrm{G}^{*}$.

Number of $\{h k l\}$ plans required and its criteria [7]

Besides the requirement to determine the six components of $\mathrm{G}^{*}$, probable measurement errors and existing experimental limitation should be taken into account. Larger the diffraction angle $2 \theta$, smaller the probable measurement errors. Thus, for a sample in Al-alloy with a FCC structure, $\{111\}$ and $\{200\}$ plans are not considered. Moreover, more $\{h k l\}$ plans are considered, more precise the calculation will be. With regard to the accessibility by the goniometer, $18\{h k l\}$ plans fulfill the requirement. Yet, the clips of the micro-tensile machine bounds an accessible angle for X-ray beam diffracting towards the sample. Even so, weak diffraction beam intensity is obtained once the cradle inclines more than $75^{\circ}$ along $\psi$-axis. Lastly, $(\psi, \varphi)_{\{h k l\}}$ located at the center is always avoided with calculation concerns. As a result, after eliminating all the inappropriate $(\psi, \varphi)_{\{h k l\}}, 12\{h k l\}$ plans are left to be analysed.

\section{XRD measuring method}

Before determining the $\varepsilon^{\mathrm{e}}$ tensor for a mechanical test, the initial $(\psi, \varphi)_{\{h k l\}}$ should be measured with high accuracy. After deformation, each modified peak position is measured in the vicinity of the initial ones. Scans along $\psi, \varphi$ and $2 \theta$ are performed one after the other in order to find the maximum intensity of the peak, this optimisation process is repeated 3 or 4 times until convergence [8]. 
The stability of the optimisation process has been tested and validated. Several sets of parameters $(\psi, \varphi$ and $2 \theta)$ in the vicinity of a given peak (Fig. 5a) have been tested as initial positions. After 4 iterations, all the processes converged to nearly the same $2 \theta$ value with a variance of $0.018^{\circ}$. The entire optimisation process for the measurement of one peak takes 4.3 minutes. For the final peak position determination for $2 \theta$, a spline function is applied for curve fitting and its maxima is taken.

Further refinement of beam size - Mosaicity

During XRD scanning, if mosaicity exists or develops, more than one peak will be found in the scanning curve. It is then difficult to determine the position of the given peak. So, the beam size has been refined to $0.1 \times 0.1 \mathrm{~mm}^{2}$ to avoid this problem. The intensity is sufficient for the peak measurement and optimisation, as the signal to noise ratio remains higher than 60 (Fig. 6).

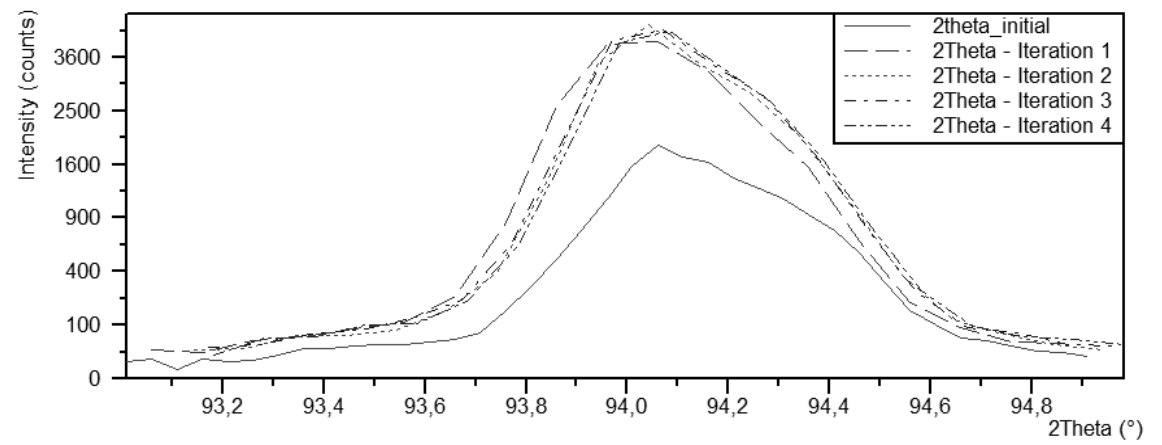

Figure 6. Grain3: Optimisation of the $2 \theta_{\{311\}}$ with beam size $0.1 \times 0.1 \mathrm{~mm}^{2}$.

\section{$\varepsilon^{t}$ field measurement}

DIC measuring method

The digital image correlation is a $2 \mathrm{D}$ or $3 \mathrm{D}$ optical method for measuring the displacement between two images of the same region of the sample taken successively. For this, a speckled pattern painting (Fig. 7a) is applied on the sample surface. During the mechanical test, images of the deformed sample surface are taken regularly. Software Correli_Q4 [9] is used to match small areas of the initial image to the corresponding ones in the deformed image and calculate the displacement field. Hence, a strain field can be derived (Fig. 7b).

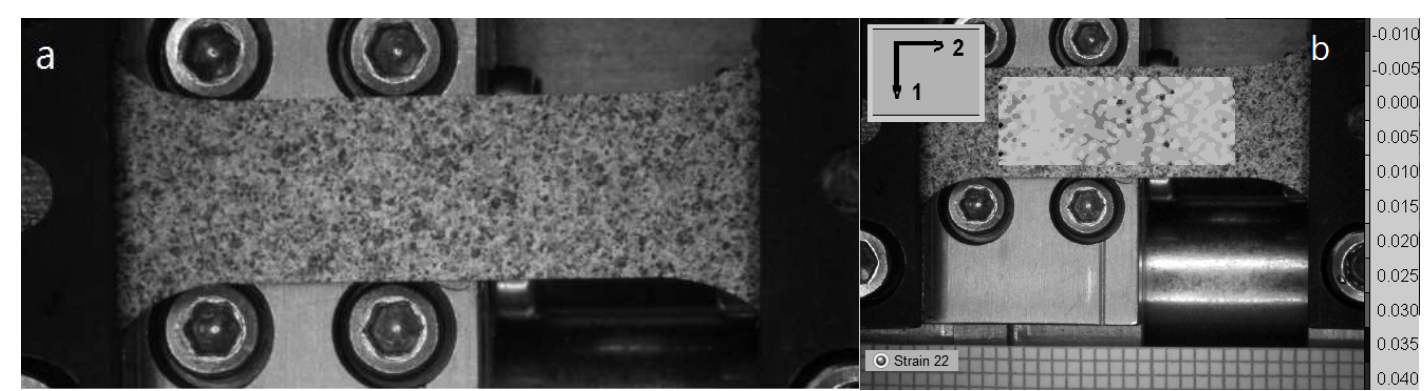

Figure 7. a. Speckle on the sample surface. b. Strain field derived by CORRELI_Q4

The speckle is made of black and white spray painting. A polycrystalline sample covered by mouchetis was tested by Correli_Q4 to qualify the painting thickness, grey histogram and measurement errors. Moreover, it was verified the speckle spread out on the surface does not bother the diffraction measurement. To ensure the painting does not contain any metallic components neither bother the XRD, comparison was made with a glass sample with the same thickness of speckle (Fig. 8). 


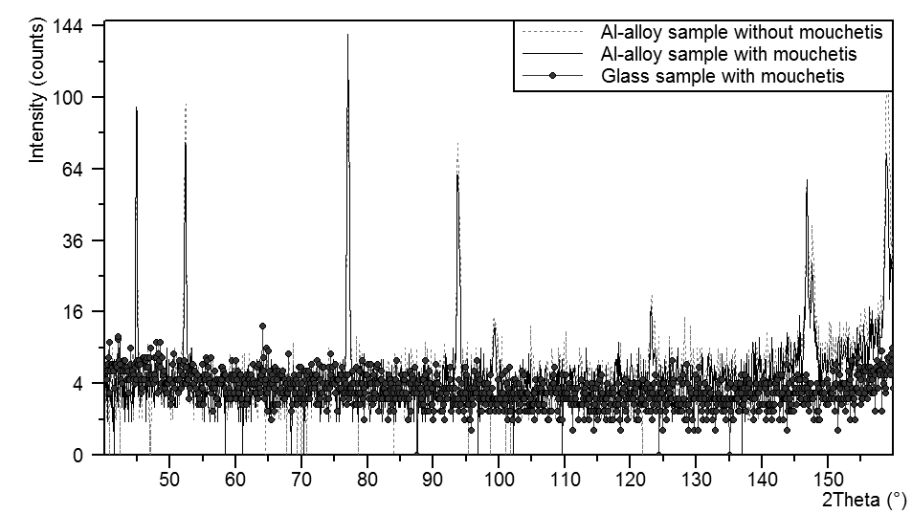

Figure 8 . Speckle verification realised by XRD measurement.

\section{Summary}

This paper presents a method developed for measuring $\varepsilon^{t}$ and $\varepsilon^{\mathrm{e}}$ strain fields at the grain scale. It introduces the device specifically developed, presents the measurement concept and describes testing procedure, analysis method as well as the precautions taken to minimise experimental errors. The experiments are still in process and result analyses will be available soon.

\section{References}

[1] Saai A., Physical model of the plasticity of a metal crystal CFC subjected to alternating loads: Contribution to the definition of multiscale modeling of shaping metal, Thesis (University of Savoie), (2008)

[2] Saai A., Louche H., Tabourot L., Chang H.J., "Experimental and numerical study of the thermo-mechanical behavior of Al bi-crystal in tension using full field measurements and micromechanical modeling", Mechanics of Materials, 42 (2010) pp. 275-292.

[3] Badulescu C., Grédiac M., Haddadi H. ,Mathias J.-D., Balandraud X., Tran H.-S., "Applying the grid method and infrared thermography to investigate plastic deformation in aluminium multicrystal", 43 (2010) pp. 36-53.

[4] P. Evrard, A. El Bartali, V. Aubin, C. Rey, S. Degallaix, D. Kondo, "Influence of boundary conditions on bi-phased polycrystal microstructure calculation", International Journal of Solids and Structures, 47 (2010) pp. 1979-1986.

[5] Avril S., Bonnet M., Bretelle A.S., Grédiac M., Hild F., Ienny P., Latourte F., Lemosse D., Pagano S., Pagnacco E., Pierron F.,"Overview of identification methods of mechanical parameters based on full-field measurement", Experimental Mechanics, 48 (2008) pp. 381-402.

[6] Ortner B., Simultaneous determination of the lattice constant and elastic strain in cubic single crystal, Adv. X-ray Analysis, 29 (1986) pp. 387-394.

[7] Ortner B., The choice of lattice planes in X-ray strain measurements of single crystals, Adv. X-ray Analysis, 29 (1986) pp. 113-118.

[8] EBERL F., Second order heterogeneities in a multicrystal: Experimental developments using X-ray Diffraction and comparison with a Finite Element Model, Thesis (ENSAM Paris), (2000)

[9] Hild F., ROUX S., CORRELIQ4: A Software for "Finite-element" Displacement Field Measurements by Digital Image Correlation, Internal report of ENS Cachan, 269, (2008) 